Schreiter, K.; Müller, S.; Luckner,R.; Manzey, D.

\title{
Demand Control Law for Total Energy Angle Tested at Manual Approaches
}

Journal article | Accepted manuscript (Postprint)

This version is available at https://doi.org/10.14279/depositonce-7844

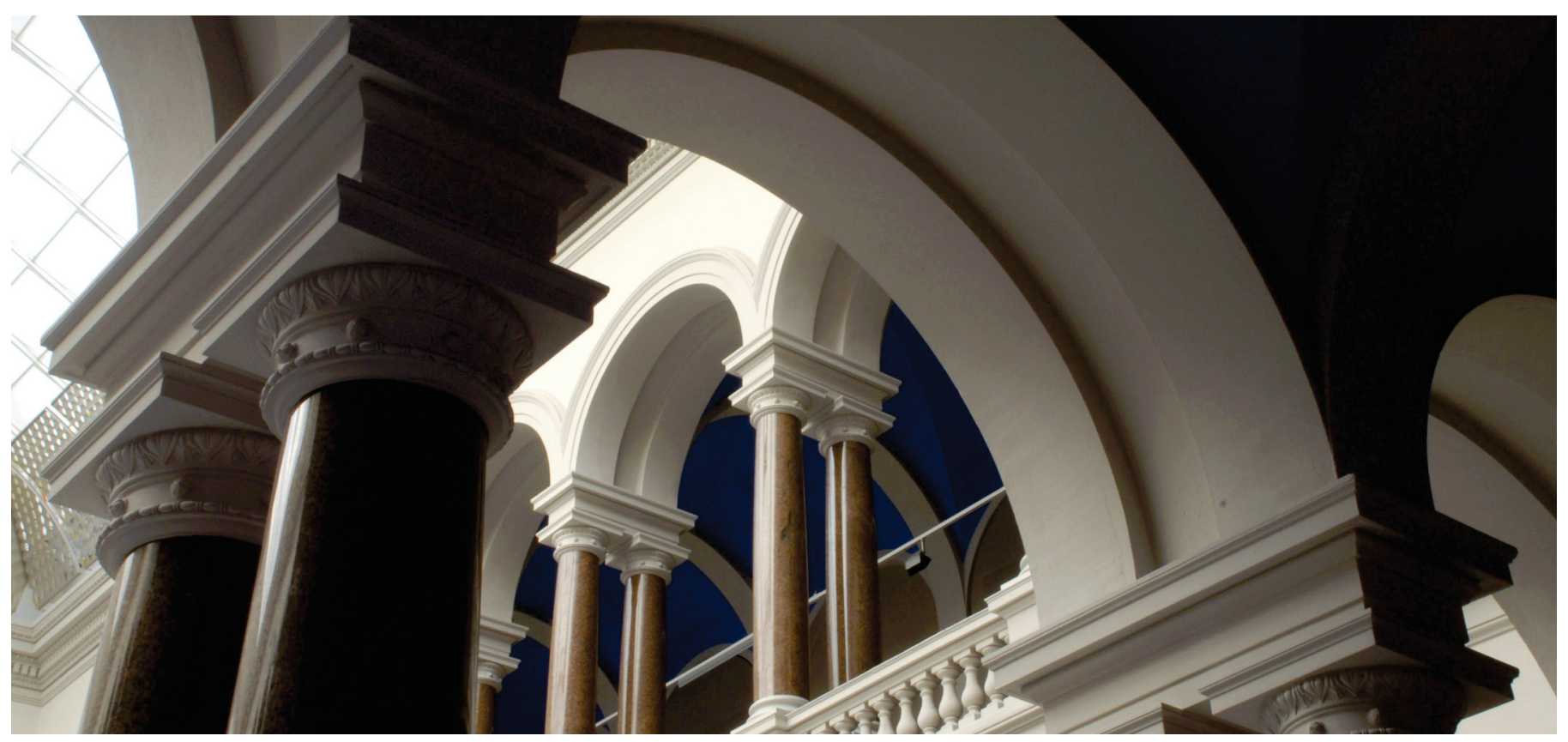

Schreiter, K., Müller, S., Luckner, R., \& Manzey, D. (2018). Demand Control Law for Total Energy Angle Tested at Manual Approaches. Journal of Guidance, Control, and Dynamics, 41(6), 1443-1448. https:// doi.org/10.2514/1.g003194 


\section{Demand Control Law for Total Energy Angle Tested at Manual Approaches}

\author{
K. Schreiter, ${ }^{*}$ S. Müller, ${ }^{\dagger}$ R. Luckner, ${ }^{\dagger}$ and D. Manzey ${ }^{\S}$ \\ Technische Universität Berlin, 10587 Berlin, Germany
}

\section{Introduction}

A IR traffic is continuously growing and reduced aircraft separations as well as more complex flight trajectories are introduced to increase capacity at congested hubs while minimizing environmental impact. Complex flight trajectories allow optimizing the use of airspace, and precise speed control enables decreasing separation distances. Fully automated flight allows meeting these emerging precision requirements. However, it is essential that pilots can take manual control at any time, either for short-term flight-path changes or in case of an autopilot failure. Furthermore, the Federal Aviation Administration (FAA) recommends that pilots should sufficiently train manual flying skills in daily operations [1]. The expected high-precision requirements of future air traffic will significantly raise the workload in manual flight. Fly-by-wire technology supports pilots during manual flight. It changes the conventional direct relation between input device and control surface deflections to the command and control of flight parameters, for example, $n_{z}$ law in Airbus aircraft [2]. The flight control laws improve handling qualities, adapt aircraft response to pilot's demands (without the need for trimming), and automatically compensate disturbances in manual flight. These characteristics can reduce workload while pilots stay in the control loop. Today's transport aircraft use this augmented manual control only for attitude control with aerodynamic control surfaces, whereas engines and speedbrakes are still used conventionally. Pilots control the energy state of the aircraft by changing thrust and spoiler lever positions. They observe fan rotation speed or engine pressure ratio (EPR) as well as spoiler deflection and adjust their inputs according to deviations from the intended vertical flight path and airspeed. As the aircraft reaction to an input depends on the actual flight state, the pilots have to anticipate this impact on their inputs. The described complex control concept generates high cognitive and motoric workload that will increase with the future precision requirements.

A concept for a flight control augmentation system concerning this gap of support systems for manual flight was described by Schreiter et al. [3] and Müller et al. [4]. The so-called nxControl system addresses commercial transport aircraft. It provides a

\footnotetext{
*Research Scientist, Flight Mechanics, Flight Control and Aeroelasticity. Member AIAA.

${ }^{\dagger}$ Research Scientist, Work, Engineering and Organizational Psychology.

†Professor, Flight Mechanics, Flight Control and Aeroelasticity. Associate Fellow AIAA.

${ }^{\S}$ Professor, Work, Engineering and Organizational Psychology.
}

control law for longitudinal load factor $n_{x}$. The pilot can directly command $n_{x}$. Engines and spoilers are regulated according to the command and the actual flight state. To the best of our knowledge, the parameter $n_{x}$ is currently not used as a control variable for manual flight of transport aircraft, neither in commercial airliners nor in research. The control law is supported by adapted additional symbols on the flight displays. These symbols allow direct control and monitoring of the energy state rates as well as the system functions. The system aims at more precise manual flight with lower workload and therefore increasing safety by enabling manual flight in daily operations under the demanding requirements of future air traffic. The nxControl system is designed to introduce only minor changes in work flow compared with conventional flight to avoid influences to the basic flying skills. In addition, it shall provide advanced awareness and control of energy state. A first nxControl prototype was evaluated in a flight simulator campaign with 11 airline pilots. It was tested with four standard flight tasks (airwork) [4] and a standard straight-in approach to runway 25C at Frankfurt (Main) [5,6]. The objective was to verify whether nxControl improves flight precision and reduces pilot workload. The pilots were able to fulfill the given tasks with nxControl with the same precision and less thrust lever movements as with conventional thrust control after only $1.5 \mathrm{~h}$ training. However, the expected significant difference in precision compared with the conventional manual flight did not occur. An explanation is that it was easy to achieve the standard accuracy for the given tasks with conventional control as well as with nxControl. Therefore, the benefits of the augmentation system could not emerge. Nevertheless, eye-tracking measurements showed a change in the scanning pattern at the displays. The pilots determined energy information from the centre of the primary flight display (PFD), where nxControl provides cues for flight path angle and total energy angle, instead of the engine warning display. Therefore, the focus of scanning has moved to the PFD.

Since then, the nxControl system has been improved. First, the control law was optimized, providing constant handling characteristics all over the flight envelope. Second, the human-machine interface (HMI) was improved with a specifically designed inceptor concept. Section II explains the nxControl system with focus on the flight mechanical background and the design process of the control laws. The display concept is briefly recapitulated (details can be found in [4]). This new prototype of the nxControl system was evaluated in a flight simulator study. The objective was to compare $\mathrm{nxC}$ Control and conventional control in a highly demanding approach pattern for Salzburg airport. This task was more challenging than the standard tasks of the prior study. The experiment design and the results of the simulator tests are described in Secs. III and IV. The novel contributions are the complete description of the nxControl system and the simulator test results that validate the advantages of the system at today's demanding trajectories.

\section{Flight Control Augmentation System nxControl}

In today's sidestick-controlled commercial airliners, the vertical load factor $n_{z}$ is used to control pitching movements in manual flight. Together with thrust, pilots control airspeed and flight-path angle. Engines are conventionally set either via fan rotation speed N1 or EPR. As an improvement, $\mathrm{nxC}$ Control uses the longitudinal load factor $n_{x}$ for computing the adequate thrust control commands in a feedback controller. With $n_{x}$, pilots command the change of total energy (sum of potential and kinetic energy). Together with the $n_{z}$ command of the sidestick, pilots decide whether the energy change is converted into flight-path angle and/or into airspeed changes. 


\section{A. Flight Mechanical Background}

The fundamental flight mechanical relationships are well established although under varying terminology. The vector of total load factor is defined as the ratio of the external forces, that is, the sum of aerodynamic forces and thrust, to weight in an arbitrary axis system [7]. It corresponds to the vector of the aircraft acceleration divided by the gravitational constant. This relation can be obtained by Newton's second law of motion for rigid aircraft with forces and mass concentrated at the center of gravity. The longitudinal load factor in flight-path direction $n_{x k \text {,tot }}$ that nxControl uses can then be derived from the drag equation (longitudinal force equation) in flight-path axis for symmetric flight, that is, zero sideslip angle, bank angle, and derivative of azimuth angle:

$$
\begin{aligned}
n_{x k, \mathrm{tot}} & =\frac{1}{W}\left[T \cos \left(\alpha+\sigma-\alpha_{W}\right)-D \cos \alpha_{W}+L \sin \alpha_{W}\right] \\
& =\frac{\dot{V}_{K}}{g}+\sin \gamma
\end{aligned}
$$

For simplified description it is assumed that the sum of thrust incidence angle $\sigma$ and angle-of-attack $\alpha$ equals zero $(\sigma+\alpha=0)$. Furthermore, the angle between airspeed and flight-path velocity due to wind $\alpha_{W}$ is small $\left(\cos \alpha_{W} \approx 1, \sin \alpha_{W} \approx \alpha_{W}\right)$. The ratio lift $L$ to weight $W$ is approximately equal to the normal load factor $n_{z k \text {,tot }}$, which is nearly 1 in steady flight conditions. These simplifications lead to

$$
n_{x k, \mathrm{tot}}=\frac{T-D}{W}+n_{z k, \mathrm{tot}} \alpha_{W}=\frac{\dot{V}_{K}}{g}+\sin \gamma
$$

So, the load factor $n_{x k \text {,tot }}$ (abbreviated as $n_{x}$ ) is either expressed by the external forces (thrust force $T$ and aerodynamic drag force $D$ related to weight $W$, wind angle of attack $\alpha_{W}$ amplified by the maneuver loads) or by flight parameters (flight-path acceleration $\dot{V}_{K}$ divided by the gravitational constant $g$, flight-path angle $\gamma$ ). The difference between thrust and drag related to weight for a constant wind is also known as specific excess thrust. A pilot can actively influence thrust and drag by commanding thrust $\delta_{T}$, spoiler deflections $\delta_{S}$, and flap deflections $\eta_{K}$, whereas wind disturbs $n_{x}$.

The relation between pilot inputs and resulting thrust and drag forces is nonlinear and depends on the actual flight state, especially airspeed and altitude. Figure 1 shows thrust and drag force qualitatively as a function of equivalent airspeed $V_{\text {EAS }}$ and their dependencies on control devices at constant altitude. If thrust equals drag, airspeed remains constant and corresponds to the intersection of the two graphs (trim point $Z_{1}$ ). With changing drag force $D$ via $\delta_{S}$ or thrust force $T$ via $\delta_{T}$, as shown in Fig. 1 by the shifted thrust curve (dashed line), the difference represents the longitudinal load factor $n_{x}$. Following Eq. (2), this difference can be used to change either airspeed or flight-path angle, or both. The first two cases are shown in Fig. 2. If the flight path is maintained (dashed line), the excess thrust accelerates the aircraft. However, drag increases with rising airspeed (if above minimum drag speed $\left.V_{\mathrm{MD}}\right)$ and thus acceleration decreases. After a long period of time (not shown in Fig. 2), the change in thrust or drag leads to a new

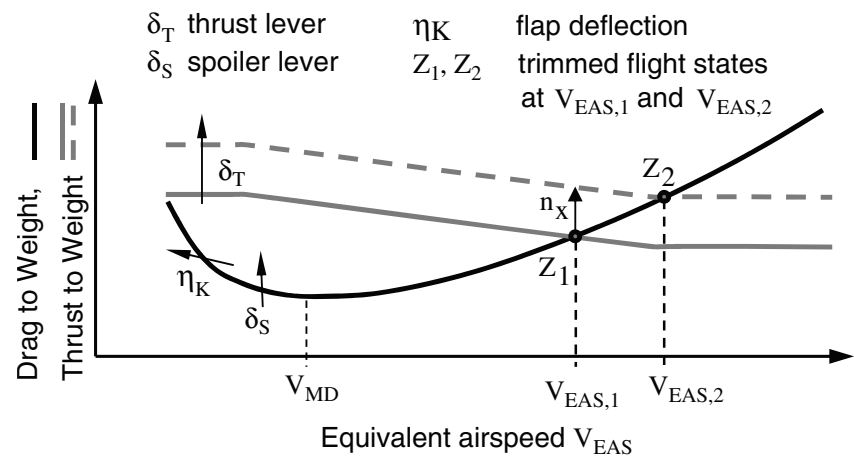

Fig. 1 Thrust and drag related to weight versus equivalent airspeed and their qualitative dependence on pilot commands.
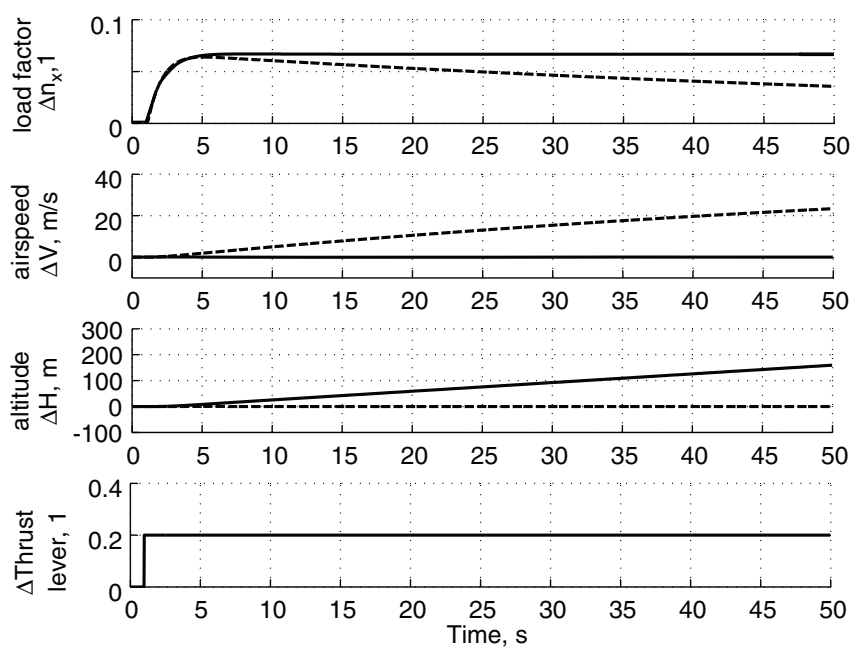

Fig. 2 Thrust lever step responses for maintained airspeed (full line) and maintained altitude (dashed line).

trimmed state $Z_{2}$. If, otherwise, the amount of $n_{x}$ is used to change altitude at constant airspeed (full line), drag does not change, excess thrust remains constant, and the aircraft climbs. As Fig. 2 shows, setting thrust and maintain altitude with elevator would be sufficient to change airspeed from state $Z_{1}$ to $Z_{2}$. For faster aircraft reaction, pilots set higher thrust changes and reduce it when the target airspeed is reached. This type of pilot command is similar to a rate command (acceleration)/attitude hold (airspeed). To change altitude at constant airspeed, pilots control climb rate with thrust and sink rate with thrust and spoilers. Airspeed is controlled indirectly by changing pitch attitude. The nonlinear speed-dependent aircraft behavior after a thrust input is difficult to anticipate for pilots, especially delays in engine reaction. The common pilot strategy is to use block inputs for thrust and spoiler levers. They command empirical values and adjust them if the aircraft reaction in airspeed or flight-path angle is not as expected. "Pitch-and-power" tables are used as an aid. They comprise precalculated combinations of thrust values (either fan rotation speed or EPR, depending on engine type) and pitch angle for steady flight conditions at different altitudes and airspeeds. However, precise thrust commands and maintaining the intended flight condition usually requires several adjustments. This process needs to be improved in order to achieve more precise manual flight with lower workload. The $\mathrm{nxC}$ Control system supports the manual flight by changing the input from conventional parameters (N1 or EPR and incremental spoiler setting) to $n_{x}$ as a meaningful flight parameter that represents the intended flight state changes. For an intuitive interpretation of the load factor $n_{x}$, the relation of acceleration and flight-path angle to the change in total energy is indicated to the pilots. This relation is described by the total energy angle $\gamma_{E}$

$$
\sin \gamma_{E}=\frac{\dot{E}_{\mathrm{tot}}}{W V_{K}}=\frac{\dot{V}_{K}}{g}+\sin \gamma
$$

The total energy angle $\gamma_{E}$ (also called total flight-path climb angle [7]) quantifies the change in total energy $\dot{E}_{\text {tot }}$ related to weight and flight-path speed in an angular value. It is equal to the load factor $n_{x}$ in Eq. (2). For manual flight, Eqs. (2) and (3) describe the ability of the pilot to change the total energy at a certain rate by setting thrust or drag. By changing the flight-path angle, the pilot can distribute the total energy rate either to potential or kinetic energy rate or to both. The total energy angle has the same unit as the flight-path angle and they can be compared directly. The difference $\left(\sin \gamma_{E}-\sin \gamma\right)$ yields the dimensionless speed derivative $\dot{V}_{K} / g$ [see Eq. (3)]. Displaying $\gamma_{E}$ as representation of $n_{x}$ allows pilots easier predicting the change in flight state induced by thrust and spoiler commands. Together with the nxController, pilots can directly command the intended change in flight state. As pilots control the energy state of an aircraft by calibrated airspeed instead of flight-path speed, the total energy angle for the nxControl system is based on calibrated airspeed. 


\section{B. Feedback Controller for the Longitudinal Load Factor}

The longitudinal load factor $n_{x}$ is the command and control variable of the nxController. It is adjusted by controlling engines and spoilers (airbrakes). As two control devices are available for one control variable, a control allocation law is necessary. Airline pilots use thrust as primary control and spoilers as secondary control to manage the energy state. They use spoilers only if the energy decrease with idle thrust is not enough and retract them before they raise thrust level. This pilot behavior is similar to a daisy chain control allocation approach, where the control effectors are sequentially used until they reach their saturation in a given hierarchy (see, e.g., [8]). As a pilotcentered design, the control allocation of nxControl is based on this pilot behavior. With the daisy chain hierarchy, the control law can switch between two separate functions for engines and spoilers (see Fig. 3). The function for engines is active if spoilers are fully retracted. The function for spoilers is active if engines are in idle. Situation awareness is achieved, as the pilot must allow the use of spoilers by a switch on the nxLever (see Fig. 4). Without activation, the spoiler function is bypassed.

Separating the two control functions allows individual controller designs for both effectors. For preliminary controller design, linear transfer functions of the aircraft dynamics between engine and spoiler inputs and the load factor $n_{x}$ response were determined. The controller design considers two step response types: constant airspeed and constant altitude. For constant airspeed, the $n_{x}$ response is described by an aperiodic low-pass system with the steady-state value $k$-for engines of third order and for spoilers of first order. However, when airspeed changes, $n_{x}$ tends to zero for $t \rightarrow \infty$ due to the change of drag (as shown in Fig. 2, dashed line). This effect is represented in the transfer function by an additional derivative element with first-order $\operatorname{lag}\left(\tau_{4}\right.$ is large). Note that the time constant of this derivative element is positive for airspeeds above minimum drag speed and negative below. So, the transfer function of thrust command to load factor $F_{n_{x}, \mathrm{THR}_{\mathrm{com}}}$ is

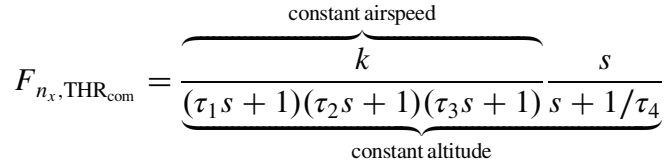

The transfer function between spoiler command and $n_{x}$ response is derived in the same way. Both transfer functions, together with the

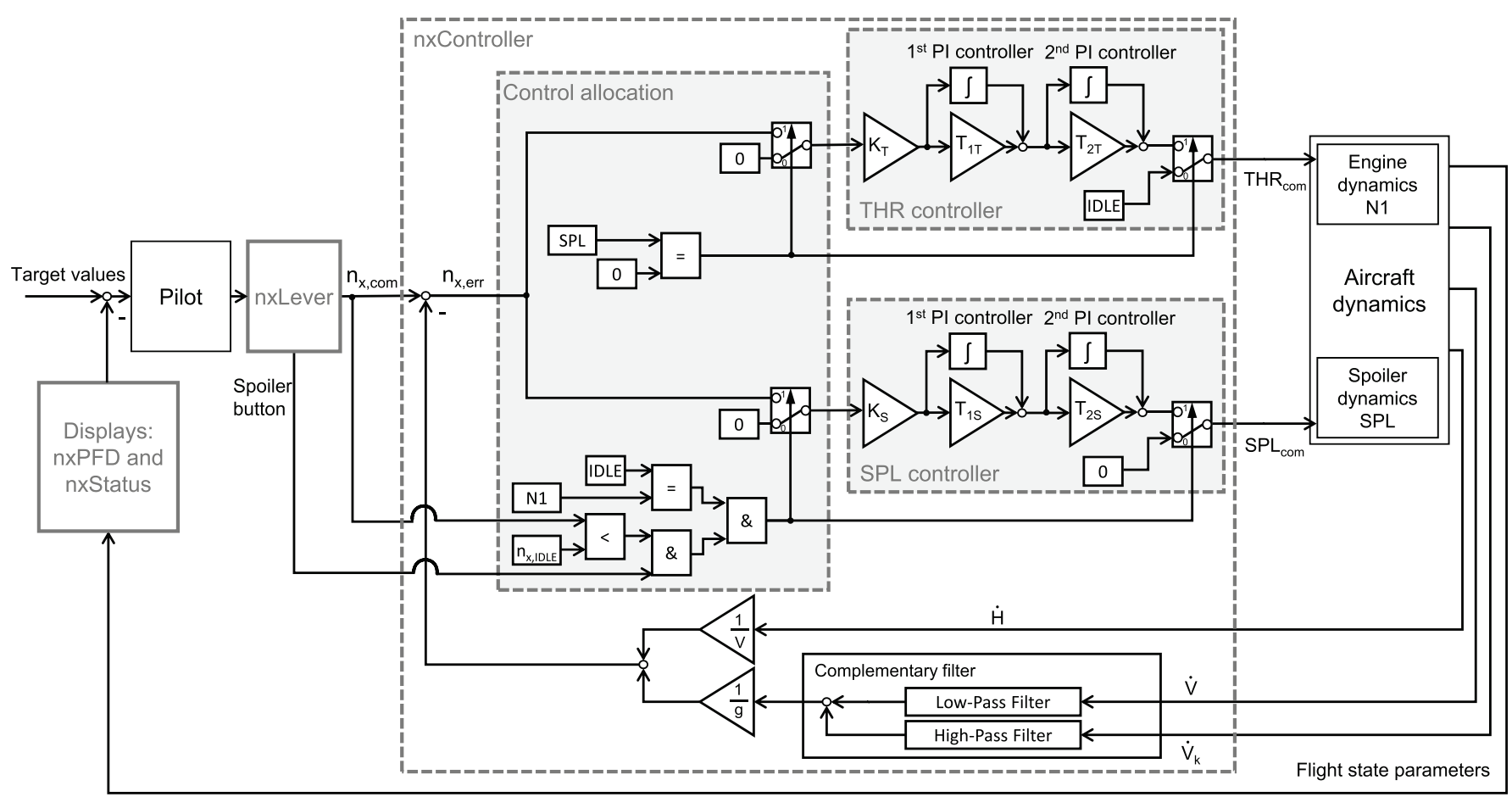

Fig. 3 Control loop of the nxControl system with pilot in the loop.

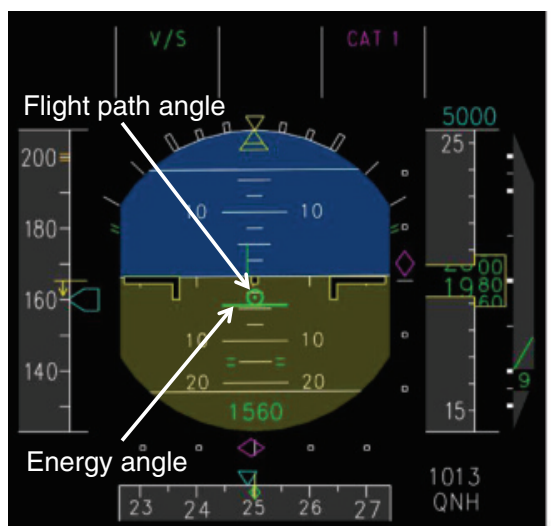

a) nxPFD: Total energy angle and flight path angle at artificial horizon

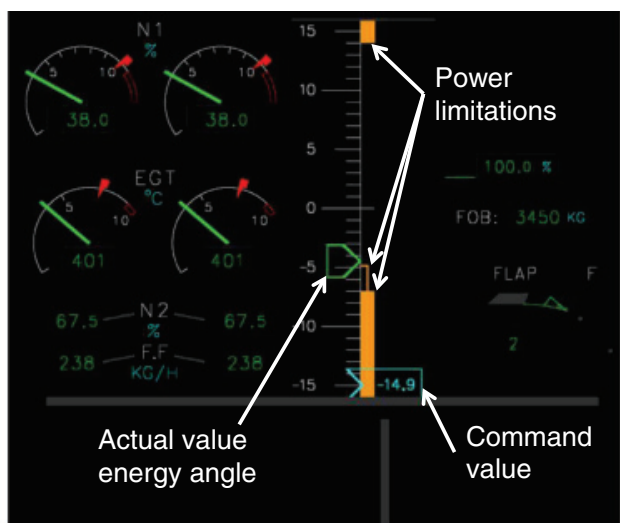

b) nxStatus at engine warning display: vertical degree scale for energy angle and command value

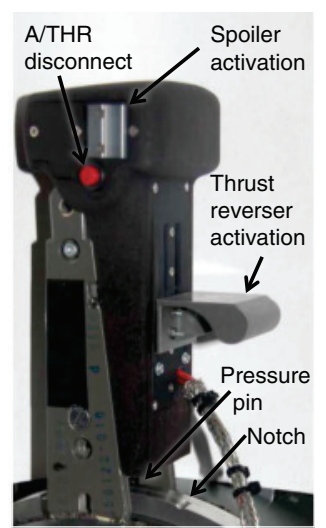

c) nxLever: handle with spoiler switch

Fig. 4 HMI of the nxControl system. 
following requirements, are the basis for the architecture of the controller. A primary requirement that pilots expect from a control system for the closed-loop input response is steady-state accuracy. Therefore, the nxController needs integrative behavior. The zero in the open-loop system (when altitude is maintained) is eliminated by a further integrator. As integrators adversely affect dynamic stability, the integrator effect is limited to low frequencies via two subsequent firstorder proportional-integral transfer functions. The thrust command caused by a load factor error $F_{\mathrm{THR}_{\mathrm{com}}, n_{\mathrm{x} \text { er }}}$ is given by the transfer function

$$
F_{\mathrm{THR}_{\mathrm{com}}, n_{x, \mathrm{err}}}=K_{T} \frac{T_{1 T} s+1}{s} \frac{T_{2 T} s+1}{s}
$$

The transfer function of the spoiler controller $F_{\mathrm{SPL}_{\mathrm{com}}, n_{n_{\text {er }}}}$ has the same structure. Figure 3 shows this architecture for both thrust and spoiler controller.

The controller gains were optimized with the software tool MultiObjective Parameter Synthesis (MOPS) [9]. Both the described linear aircraft model and a highly sophisticated nonlinear simulation model were implemented in MOPS. A set of optimization criteria in the time and frequency domain was set up as the so-called bad/good criteria. The results of preliminary studies, standard controller requirements, and model analysis were used to define the criteria parameter. Most of the requirements for longitudinal flying and handling qualities, such as $\mathrm{C}^{\star}$-criterion [10], consider only the pitch dynamics in the frequency band of the short period mode. Only a few exist for the range of flight-path dynamics that is relevant for the nxControl system. Therefore, suitable requirements in the frequency domain, such as damping ratios and time delay margin, and in the time domain, such as rise time, overshoot, steady-state offset, maximum error, and mean error of the control variable, were defined to achieve a response behavior in the limits that are shown in Fig. 5. This behavior was applied to the whole flight envelope by gain-scheduling with airspeed and altitude. Starting with a gain set optimized in the center of the flight envelope, the gain schedule was optimized for selected envelope points. The results are step responses that are nearly independent from flight condition.

Figure 6 compares step responses when altitude is maintained for $n_{x}$ command with nxControl and the response to a thrust step input that results in the same initial $n_{x}$ value of 0.1 . Altitude was maintained in both cases as this case is more demanding regarding steady-state accuracy. It is apparent that in the conventional case an $n_{x}$ reduction occurs at rising airspeeds. This error is eliminated by the nxController by increasing fan rotation speed. As a result, the acceleration is constant and allows for precise airspeed setting. The step response for constant airspeed (not shown) is qualitatively similar to the conventional response as thrust adjustments are negligible. The

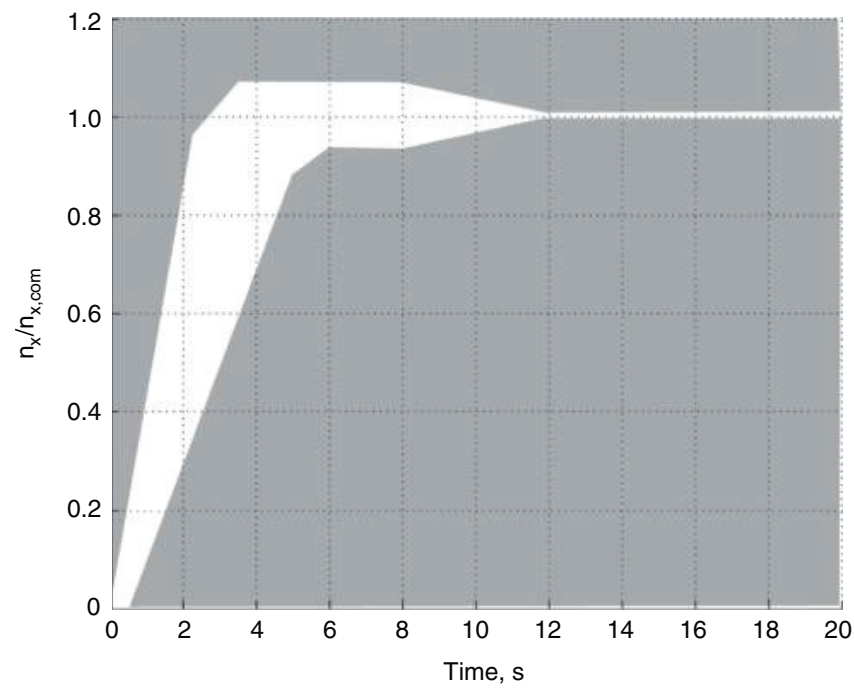

Fig. 5 Design boundaries for $n_{x}$ time response.
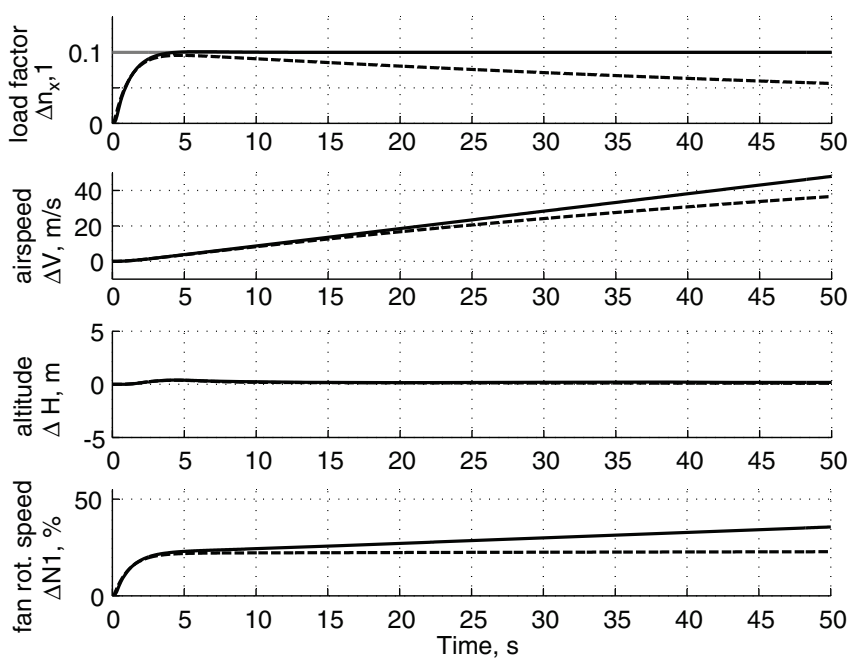

Fig. 6 Step response to command $\Delta n_{x, \text { com }}$ (gray line) with nxController (full line) and conventional thrust command (dashed line).

advantage of nxControl is that pilots do not have to iterate for the correct fan rotation speed that fits to the required flight-path angle.

Because $n_{x}$ is calculated from airspeed and flight-path angle, every disturbance (e.g., changes in aircraft configuration or wind) is immediately corrected by the controller without pilot action. So, the pilot workload is reduced. However, the influence of high-frequency turbulence on thrust control needs to be reduced as it would negatively affect engine life. Therefore, airspeed is filtered with thirdorder low pass. The high-frequency information that is needed for energy angle control is retrieved from flight-path speed by a thirdorder high-pass filter for flight-path speed.

\section{HMI for Controller and Visualization of Energy Angle}

Three new elements were added to the standard cockpit layout (see Fig. 4). First, a symbol for the total energy angle (horizontal line) was integrated on the PFD. It is linked to the pitch scale and allows tracing of energy changes. The modified PFD is called nxPFD (see Fig. 4a). The "birdy" as a common symbol for flight-path vector was changed to a circle with a center dot that indicates flight-path angle without drift information. The flight-path angle correlates to the change in altitude. The difference between $\gamma_{E}$ and $\gamma$ represents the change in airspeed in a direct manner and central on the artificial horizon. These symbols show the current state and are usable even without nxController. Similar concepts of implementing energy information to the cockpit have been introduced, for instance by Amelink et al. [11] and Lambregts et al. [12], and are used in some head-up displays in the form of specific excess thrust [13].

The nxStatus display as visual interface to the nxController is placed on the engine warning display (EWD) as shown in Fig. 4b. On a vertical degree scale, the commanded control value $\gamma_{E}$ (blue flag), the actual value (green marker), as well as the upper and lower limits for engines thrust and spoilers are depicted. The limits represent the performance envelope (minimum $\gamma_{E \text {,min }}$ and maximum $\gamma_{E \text {,max }}$ ) at a certain flight state. The limits change depending on airspeed, altitude, and aircraft configuration. Two lower limits are 1) $\gamma_{E \text {,min }}$ at idle thrust (hollow lower strip) and 2) $\gamma_{E \text {,min }}$ at idle thrust with spoilers fully extracted (filled lower strip). As the controller uses the spoilers only if the pilot has activated them, the actual value of $\gamma_{E}$ cannot fall below the idle thrust limit before the activation. Figure $4 \mathrm{~b}$ shows a situation where the pilot commands a $\gamma_{E}$ value that lies below the lower limits. The command value is at the lower end of the scale. The actual value marks $\gamma_{E \text {,min }}$ with idle thrust, because the pilot did not allow spoiler activation and a lower value is physically impossible. The upper limit marks $\gamma_{E \text {,max }}$ given by maximum thrust (take-off/go-around thrust TOGA). Concepts for scales representing thrust limits as well as command and actual value were patented by Artini et al. [14] and integrated into the PFD by Wyatt [15]. 
The inceptor for the nxController is called nxLever. In a standard cockpit, one thrust lever is installed for each engine. As the nxLever issues one command value to all engines and spoilers, one lever is sufficient. It replaces the thrust levers and the spoiler lever. Figure $4 \mathrm{c}$ shows a prototype nxLever with the additional devices for auto thrust disconnect and thrust reverser as well as a sliding switch for activation of spoilers. The lever movement is similar to the conventional thrust lever. It has a notch at the middle position in which the pressure pin on the handle bottom latches in. In this position, the $n_{x}$ command is zero, which means no energy change and maintaining the current total energy state. Above and below, energy changes are commanded linearly to the lever position. If a command for energy change is below the value that is realizable at idle thrust, the spoiler switch can be slid backward. This trigger allows the use of spoilers to adjust the control value. The switch flips forward automatically if thrust is used for control. Then the spoiler activation has to be renewed. Questions regarding the replacement of the traditional control devices by the nxLever, for example, behavior at take-off and landing or in case of engine failure, are currently investigated.

\section{Evaluation Study}

The prototype of the nxControl system was evaluated in a simulator campaign with airline pilots using the same hypotheses as in the previous study [4]. Hypothesis $\mathrm{H \# 1}$ is that nxControl allows a more precise control of longitudinal acceleration and with this a better tracking of the flight path. Hypothesis $\mathrm{H \# 2}$ is that nxControl relieves the pilots of frequent thrust adjustments and therefore lowers cognitive and motoric workload in manual flight compared with conventional manual flight. As the system was previously tested at rather simple standard tasks, a more challenging and demanding flight task was chosen for the study. The pilots had to fly an approach pattern to Salzburg airport under required navigation performance (RNP) conditions through the mountainous surrounding, with steep glide slope, and wind disturbance. It was expected that such a complex task should emerge the advantages of the nxControl system as compared with conventional pitch-and-power flying. The experiments were conducted in the fixed-base research flight simulator Simulator for Educational Projects and Highly Innovative Research (SEPHIR) [16]. The simulator cockpit is equipped with displays and sidestick (including control laws) similar to an Airbus aircraft. A sample of 24 male certified commercial airline pilots with Airbus-type ratings (A320: $N=20$; A330/A340: $N=3$; A380: $N=1$ ) participated. The 10 captains, 2 senior first officers, and 12 first officers were aged between 24 and 63 years, with a mean age of 40 years $(\mathrm{SD}=12.6 \mathrm{a})$. They had an average flight time of $8505 \mathrm{~h}$ $(\mathrm{SD}=7422.4 \mathrm{~h}$, range $600-25,000 \mathrm{~h})$.

\section{A. Flight Scenario and Procedure}

An existing RNP approach pattern to Salzburg runway SZG33 [17] was used. The terrain on both sides requires to fly two turns and to follow a steeper glide slope $\left(3.6^{\circ}\right)$ than usual approaches. The required performance of RNP 0.3 was tightened for the study to RNP 0.1 in order to further increase the already high demands on energy management. The lateral and vertical deviations to the target flight path were displayed using the available rhombuses and dots of the Instrumented Landing System (ILS) in the PFD. One dot lateral marked a deviation of $0.1 \mathrm{NM}$ and one dot vertical a deviation of $100 \mathrm{ft}$. Additionally, the target track angles were provided at the heading tape of the PFD. The pilots were instructed to perform the flight as precisely as possible.

Figure $7 \mathrm{a}$ shows the vertical profile together with target track angles as well as the points where aircraft configuration and speed had to be changed according to the lateral distance to the next waypoint. To reduce individual variation in lateral flight guidance, all given waypoints had to be used as fly-over waypoints instead of fly-by waypoints usually used for that approach. The turns introduce disturbances in energy management as lift and drag change with rising and decreasing bank angle. Therefore, they raise the workload of the pilots. The pilots had to follow a flight procedure that was adapted to the experimental aim as accurately as possible. The procedure increased the requirements to manual flight even more, as the glide slope had to be intercepted with rather high speed in clean configuration at $12,000 \mathrm{ft}$ above mean sea level (MSL). Additionally the stepwise configuration of the aircraft kept the workload of the pilots high until shortly before the end of the approach. The procedure started with a straight flight segment with speed and configuration changes up to waypoint WP2. A left turn to WP3 with configuration change followed and after a short straight middle section a second turn to the right with another configuration change was initiated at WP4. The last part until decision height at $2550 \mathrm{ft}$ MSL included a straight flight segment with several speed and configuration changes, which was finished by a short right turn to runway direction at WP6. All deceleration phases had to be initiated at the given points at the flight path and performed with idle thrust. Speedbrakes were recommended for the decelerations to 175 and $145 \mathrm{kt}$. To force the use of flight instruments (head down) the visibility range of the outside view was decreased by mist. The runway came in sight shortly before decision height. A steady wind of $15 \mathrm{kt}$ from $57^{\circ}$ without turbulence disturbed the flight. As the relative wind direction changed from crosswind to tailwind at the middle section, the wind significantly interferes with the energy management. For technical reasons, all the participants sat in the captain's seat, irrespective of their usual position.

\section{B. System Configurations and Measures}

Every pilot had to perform the given task with three system configurations. Conventional configuration (Conv) is equipped with standard PFD without energy information and conventional thrust control via N1 command. Conv is the baseline representing the cockpit configuration of today's civil transport aircraft. In the $n x P F D$ configuration, energy information is added at the PFD (see Fig. 4a) and conventional thrust control via N1 command is used. nxPFD is an intermediate state of the nxControl system. It allows determining which effects can be attributed to the energy information. The configuration nxControl contains the changes of nxPFD, the additional energy information at the EWD (see Fig. 4b), and supported thrust control via nxController with energy angle command at the nxLever (see Fig. 4c). nxControl represents the complete system determining the combined influences of HMI and controller. The sequence of system configurations was balanced across the pilots (Latin square balancing) to control for effects of fatigue or training. Furthermore, each configuration was repeated twice to average the results of both trials.

To assess the effects of nxControl and nxPFD on flight-path precision, the following flight parameters representing energy management were used for comparison: airspeed, altitude, flightpath angle, and energy angle. As the procedure required certain flight-path and speed targets, the precision was measured by the deviations of these flight parameters from their target values represented by the root mean square errors (RMSE). The effect on workload was investigated with subjective questionnaires and objective measurements of lever movements. After each landing, the participants had to rate their subjective workload on the subscales of the NASA Task Load Index (TLX): mental demand, physical demand, temporal demand, performance, effort, and frustration [18]. The overall workload score was achieved without weighting the scales against each other as this was found to have a negligible effect on the results [19]. To assess how much effort the pilots had to invest in thrust control, thrust lever movements were recorded throughout the flight simulation. A lever movement was defined as a change in lever position higher than a threshold of $0.5 \%$ of the lever range (corresponding to $0.2 \mathrm{~cm}$ at the lever top) in a time interval of $2 \mathrm{~s}$. The lever activity was defined as the sum of these lever movements divided by the number of intervals. The lever activity is assumed to be correlated to the cognitive and physical workload related to thrust control. In addition, a subsequent questionnaire concerning the scanning pattern of the display information was conducted after all flight scenarios. It was asked how the nxControl system did change the scanning behavior for the display elements fan rotation speed N1, 


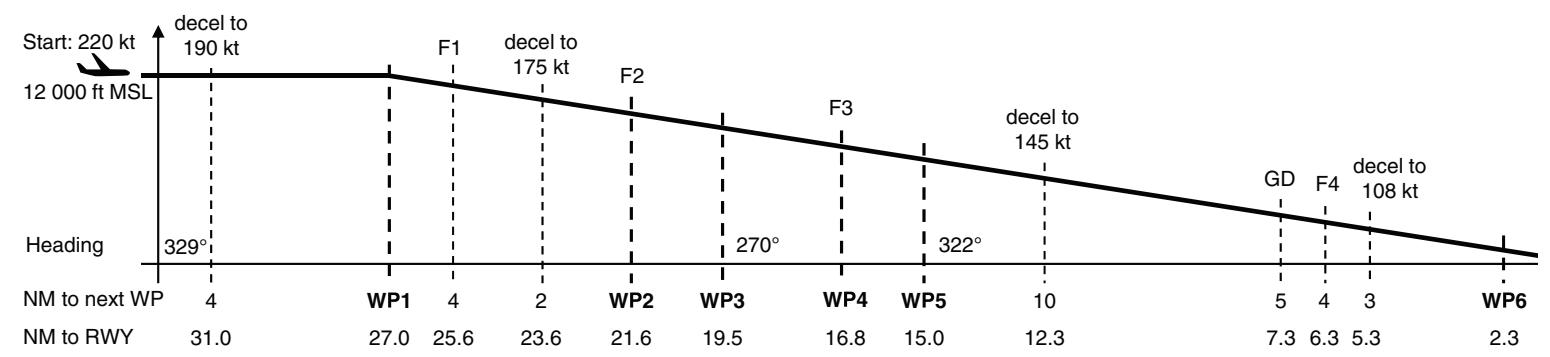

a) Procedure on vertical path to runway

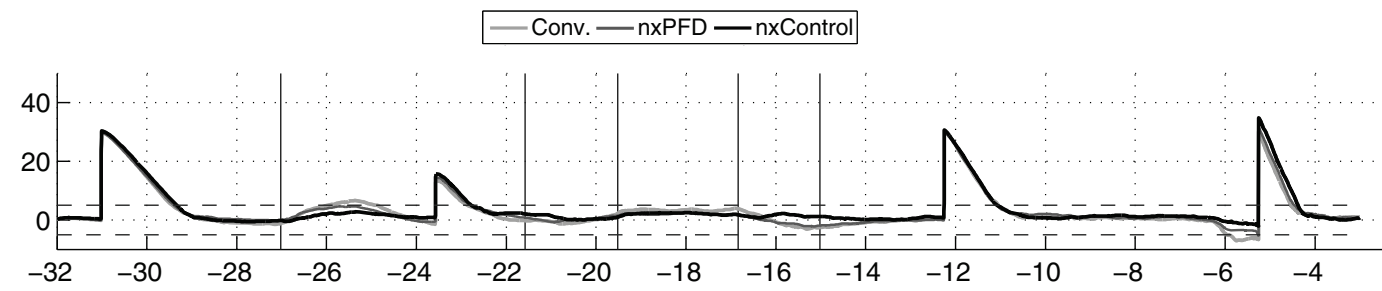

b) Speed deviation in knots against distance to runway in NM

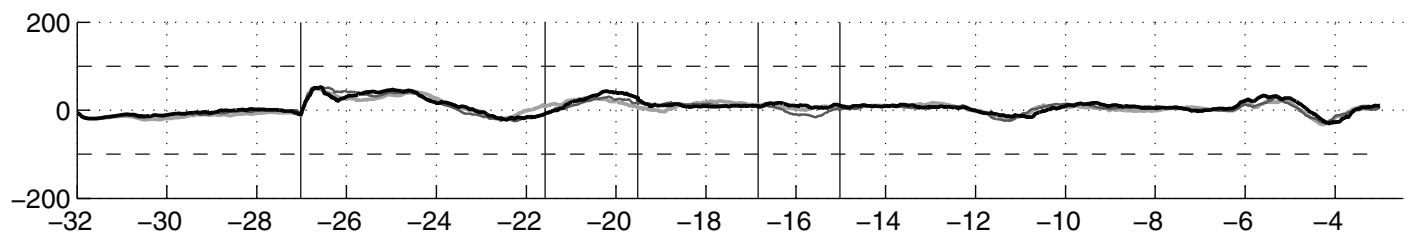

c) Altitude deviation in feet against distance to runway in NM

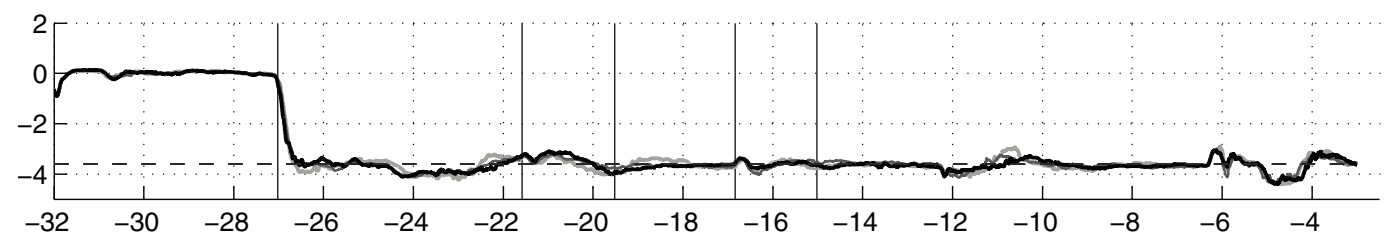

d) Flight-path angle in degrees against distance to runway in NM

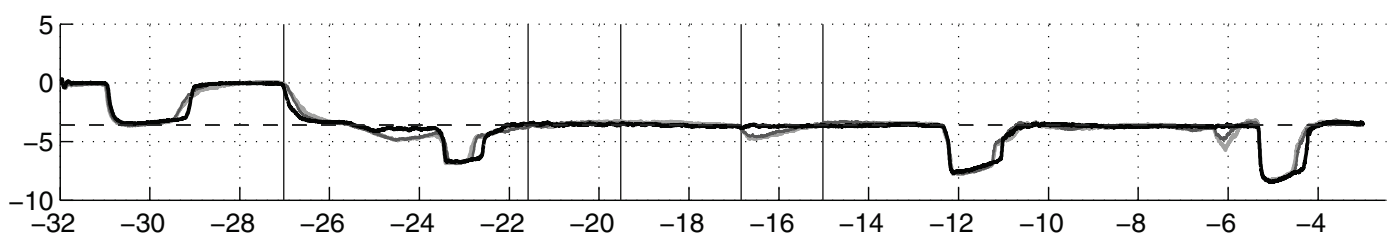

e) Energy angle in degrees against distance to run way in NM

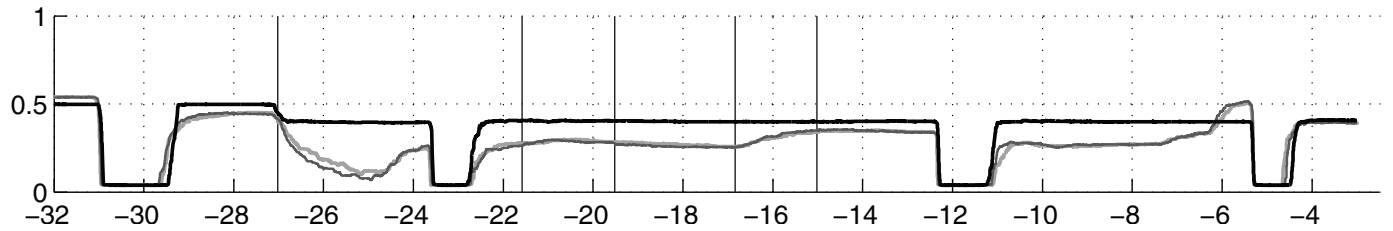

f) Normalized thrust lever position against distance to run way in NM

Fig. 7 Nominal vertical trajectory with waypoints (a) and median histories of flight parameters averaging all participants per system configuration.

pitch attitude, PFD center, barometric altitude, vertical speed, airspeed, and heading. For each element, the pilots could choose between less frequent, unchanged, and more frequent. The questionnaire was conducted to support the findings of the earlier study with eye tracking by subjective data.

\section{Results}

The results for the overall flight are shown as boxplots in Fig. 8, showing 25 and $75 \%$ percentiles, median values, and mean values (as asterisk). The data of the system configurations were analyzed pairwise in $t$-tests with multiple-testing corrections according to Śidák [20]. The $p$-values (probability of random data distributions) are given in the diagram titles. $p$-Values below the common significance level of 0.05 corroborate the statistical significance of the different mean values.

The time histories of precision and workload parameters are compared in Fig. 7. To visualize the effect of the different system configurations, the median of each parameter per system configuration is plotted against the distance to runway. The interval contains the beginning of the scenario at $32 \mathrm{NM}$ to the decision height at 3 NM. Heading was changed twice, at WP3 and WP5. 


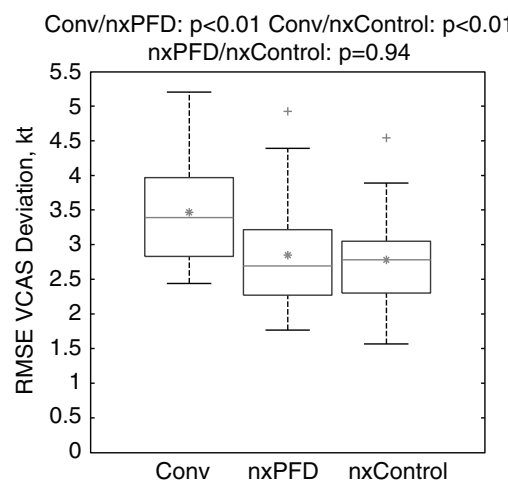

a) RMSE of airspeed

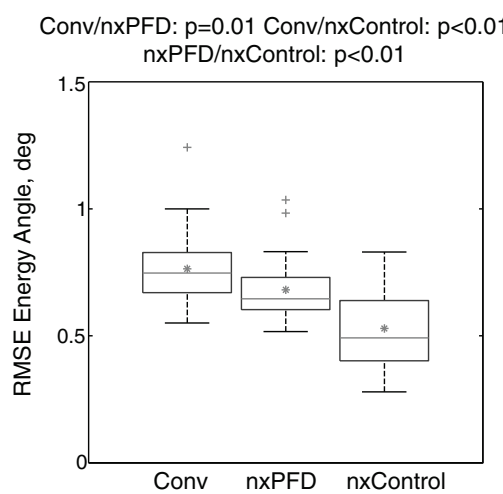

d) RMSE of energy angle

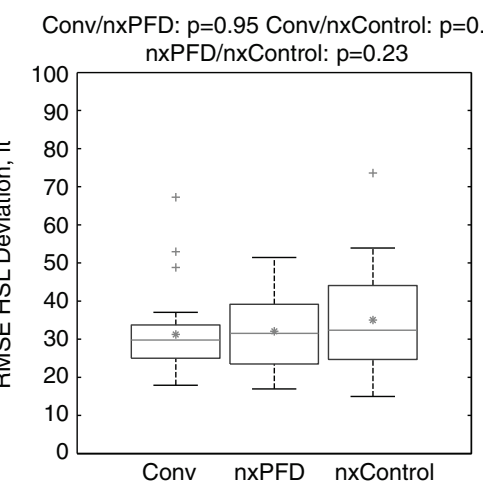

b) RMSE of altitude

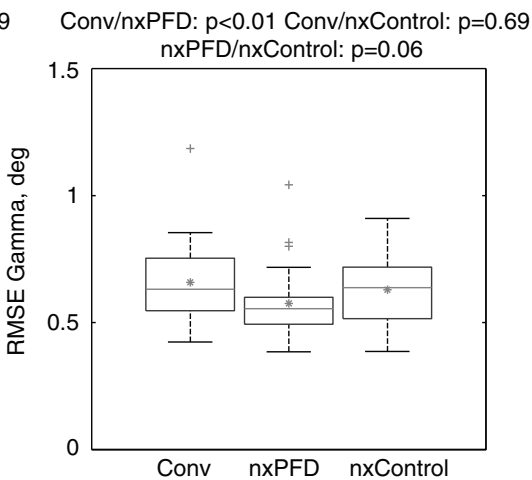

c) RMSE of flight-path angle
Conv/nxPFD: $p=0.30$ Conv/nxControl: $p<0.01$

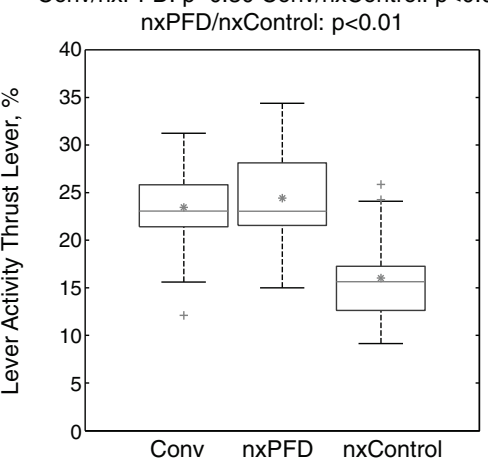

e) Lever activity
Conv/nxPFD: $p=0.03$ Conv/nxControl: $p=0.06$ nxPFD/nxControl: $p=1.00$

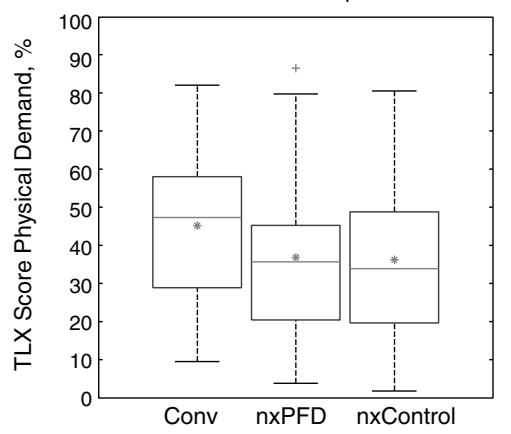

f) TLX score physical demand

Fig. 8 Statistical analysis via boxplots and means: RMSE values of precision and workload parameters.

\section{A. Flight Precision}

The boxplots in Figs. 8a-8d show the RMSE of the precision parameters. As expected, the RMSE of airspeed was significantly lower when flying with nxPFD and nxControl compared with the conventional configuration. However, a comparable effect on the RMSE of altitude was not found (no significant difference between all configurations). Results for the two additional indicators in the nxPFD revealed diverging effects. While the RMSE for the flightpath angle only benefited from nxPFD configuration, the already significantly lower RMSE for the energy angle with nxPFD was further reduced by nxControl.

The median time histories in Figs. 7b-7e show the same precision parameters. The airspeed is depicted as speed deviation from the target airspeed (Fig. 7b), which is why the plot leaps at the beginning of a commanded airspeed change and gradually decreases afterward. Analogous to the statistical analysis of the RMSE, the time history shows a higher speed deviation in the conventional configuration compared with nxPFD and nxControl at several segments. First of all, it becomes obvious that the tolerance of $\pm 5 \mathrm{kt}$ was exceeded twice in the conventional case - after glide slope (GS) intercept and after flaps configuration to full (F4). These effects were eliminated by nxPFD, and nxControl lowers the deviation at these segments even more. At the ends of the speed change segments (speed deviation lower than $5 \mathrm{kt}$ ), it can be seen that steady flight is reached faster with nxControl, compared with both other configurations. However, slight constant speed deviations (1-3 kt) are observable with nxControl (especially at the second airspeed reduction), which explains the equal RMSE compared with nxPFD. Energy disturbances, caused by the change in wind direction between WP3 and WP4 as well as by flap configuration F3 and F4, have lower negative impacts on the speed deviations in nxControl than in nxPFD or the conventional configuration.

The altitude deviation, shown in Fig. 7c, confirms the statistical comparison. There are no evident differences between the three system configurations. The time history of flight-path angle (see Fig. 7d) also shows few differences between the system configurations. At GS intercept, the pilots reached smaller deviations from the target value with nxPFD and nxControl than in the conventional configuration. Additionally, at the second airspeed change (at approx. 23-22 NM) and third airspeed change (at approx. 12-10.5 NM), the deviations from the target value are lower in both $\mathrm{nx}$ configurations. However, at the turn between WP2 and WP3, where several tasks (deceleration with spoilers, flap configuration to F2, and initiation of the turn) overlapped, nxControl shows the largest deviations of all system configurations. Nevertheless, the following less demanding segments again show a lower deviation for the $n x$-configurations.

In contrast, the time history of the energy angle in Fig. 7e shows obvious differences between the system configurations. Overall, lower variances can be identified for nxControl, which is most obvious when the flap configuration is changed to F1, F3, and F4. The nxPFD configuration only lowers the deviation at flap configuration F4. Additionally, the energy change at GS intercept was faster and more directly performed in nxControl than in both other system configurations. The disturbance by wind change had only a slight effect on the energy angle in all configurations, although the most constant trend is visible at the nxControl configuration.

\section{B. Workload}

As expected, the lever activity decreases significantly with $n x C o n t r o l$ in contrast to the conventional and $\mathrm{nxPFD}$ configurations (see Fig. 8e). Note that the use of the spoiler lever (not necessary with nxControl) is not included to the workload statistics in Fig. 8e. The subjective TLX questionnaire shows a slightly different picture. While the overall TLX score does not change significantly between the three system configurations (not illustrated), the subscale of physical demand identifies a lower value for nxPFD and nxControl, compared with the conventional configuration (see Fig. 8f).

In Fig. $7 \mathrm{f}$ the time history of the lever positions is shown. Note that with nxControl the lever position means an $n_{x}$ command to the engines, whereas in the other configurations it is a N1 command. The time histories show clearly that the pilots made fewer movements with nxLever to achieve the required flight-path and airspeed 
precision. Especially at the segments of energetic disturbances, where the controller automatically compensates, no pilot input was necessary. Fewer lever movements become especially apparent when flaps are extended and the wind changes (WP3 to WP4). In contrast, either in $\mathrm{nxPFD}$ or in the conventional configuration the pilots needed to tune the thrust setting manually with small lever position changes. Clear differences also emerged in case of required airspeed reductions. To adjust the new target airspeed, faster, less, and more direct inputs could be made with the nxLever, while multiple incremental step inputs were required by conventional thrust control until the best position is found. Also at GS intercept, a defined lever movement was recognizable for nxControl, whereas for nxPFD and conventional configuration the multiple incremental inputs for the correct lever position can be observed.

The results of the scanning questionnaire are summarized as absolute frequency of the answers less frequent I unchanged I more frequent: fan rotation speed $21|3| 0$, pitch attitude $13|10| 1$, PFD center $2|11| 11$, barometric altitude $8|15| 1$, vertical speed $15|7| 2$, airspeed $6|14| 4$, and heading $1|21| 2$.

\section{Discussion}

The results show positive effects of the different components of the nxControl system. Control of flight path and airspeed during the approach is more precise. The pilot effort in thrust control is lower. The precision in altitude is comparable to conventional thrust control. Most likely this is due to the flight-path stability provided by the $n_{z}$ flight control law of the sidestick. The $n_{z}$ law transfers changes in energy to airspeed changes and flight path is maintained. The $\mathrm{nxC}$ Control system does not influence this behavior. Since all energy changes affect mainly the airspeed precision, the effects of nxPFD and nxControl can be observed in airspeed and total energy parameters. Obviously, the centralized information on the nxPFD makes it possible to capture unintended changes in airspeed faster and more precisely than in the conventional case. This advantage may be due to the delayed reaction of the speed trend vector at the conventional airspeed indicator that shows changes only above certain thresholds (appears when greater than 2 knots and disappears when less than 1 knot per $10 \mathrm{~s}$ ). The additional energy information leads to faster recognition of errors and pilots can react more quickly to maintain the energy state with the nxControl system. Furthermore, the nxController prevents errors in energy state. Therefore, the simulation results support Hypothesis $\mathrm{H \# 1}$. A more complex picture emerged for the workload results. As expected, the objective parameter of lever activity was decreased significantly with nxControl. Especially at situations with energetic disturbances, fewer lever movements were necessary. Additionally, the inputs seem to be more goal-oriented as the command value is visible at the display and can be directly commanded with the lever position. However, on the subjective level, pilots perceived a lower physical demand only using nxPFD. But, in fact, lever activity did not change compared with conventional flying just by adding energy information at the displays. The reason for this subjective perception is not clear. It is assumed that it was caused by the pilots' ability to find the correct thrust setting more targeted with the shown energy angle in the nxPFD in contrast to the "blind searching" for the right fan rotation speed in the conventional case. The overall NASA TLX scores and all subscales (except physical demand) did not show a significant difference between conventional control, nxPFD, and nxControl, which means that the subjectively perceived overall workload did not differ between the three conditions. However, some sections of the scenario pointed out the lack of training with the new nxControl system. For example, the turn between WP2 and WP3 combining different tasks like deceleration, flap configuration, and initiation of the turn, showed higher deviations with $\mathrm{nxControl}$. It is assumed that at this point the temporal coordination of the different tasks was more demanding with the new system. Considering the confrontation with a fully new and unknown control system and the rather short time for training, a higher workload compared with the well-known and routinely used conventional system is not surprising. Additionally, the results could be affected by the unusual scanning pattern or motoric behavior of pilots who usually operate from the right-hand cockpit seat. Given this, the fact that the new system obviously had not elevated the workload can be taken as an indication that the nxControl concept can be easily learned and understood. Thus, hypothesis $\mathrm{H \# 2}$ can be confirmed for physical workload. For cognitive workload, it initially has to be rejected, but this may change with more training of nxControl. The scanning questionnaire mirrors the main findings of the prior eye-tracking study, showing that the focus of scanning shifts to the center of the PFD and that parameters like pitch and engine state are replaced by the parameters flight-path angle and total energy angle.

\section{Conclusions}

The proposed nxControl system aims to enable a more precise manual flight along highly demanding trajectories at lower workload compared with today's manual flight. The evaluation of the system in a flight simulator campaign with airline pilots shows that the overall goal was achieved. With the nxControl system, pilots were able to follow a demanding curved approach with required navigation performance RNP 0.1 with smaller mean errors in airspeed and total energy angle coupled with lower physical activity. Therefore, the results confirm the findings of precursor studies and show further positive effects of the nxControl system to precision and workload at today's demanding approach trajectories. The results indicate that $\mathrm{nxControl}$ can enable pilots to fulfill the flight-path requirements of future air traffic in manual flight with lower workload than today. Furthermore, nxControl will allow manual flight more frequently as it is recommended by authorities to maintain flying skills. The lever movements with nxControl are similar to those of a conventional thrust lever. Thus, the standard control strategy of the pilots is only marginally affected. However, by automatically compensating energy disturbances, such as wind or flap deployment, nxControl simplifies the standard control strategy. The supplementary information on the PFD and EWD change the scanning pattern. Physical flight-path parameters like flight-path angle and total energy angle are used instead of basic parameters like pitch and fan rotation speed. How these changes affect the cockpit work flow should be critically scrutinized, especially in failure conditions of subsystems, for example, engine failure. In addition, the degradation to conventional thrust control, if the nxControl function is lost, is a critical issue. In summary, the simulator results provide evidence that nxControl has the potential to enhance both the precision and safety of manual flight. Further studies are planned to determine whether the system is capable to enhance manual flight at future trajectories as well as to investigate safety critical failures like engine failure. In addition, the controller logic shall be completed by including thrust reversers and wheel brakes for take-off and landing.

\section{Acknowledgments}

This work is funded by the DFG (German Research Foundation) under contract LU 1397/3-1, MA 3759/3-1. The authors thank the participating pilots for their support.

\section{References}

[1] Federal Aviation Administration (FAA), "Safety Alert for Operators, Manual Flight Operations, SAFO 13002," 2013, http://www.faa.gov/ other_visit/aviation_industry/airline_operators/airline_safety/safo/ all_safos/media/2013/SAFO13002.pdf [retrieved 15 Aug. 2017].

[2] Favre, C., "Fly-by-Wire for Commercial Aircraft: The Airbus Experience," International Journal of Control, Vol. 59, No. 1, 1994, pp. $139-157$. doi:10.1080/00207179408923072

[3] Schreiter, K., Müller, S., and Luckner, R., "nxControl: Konzept zur Vorgaberegelung für die Längsbeschleunigung des Flugzeugs," D. Luftund Raumfahrtkongress, Deutsche Gesellschaft für Luft- und Raumfahrt, Bonn, 2013, urn:nbn:de:101:1-2013112216214.

[4] Müller, S., Schreiter, K., Manzey, D., and Luckner, R., "nxControl Instead of Pitch-and-Power: A Concept for Enhanced Manual Flight Control," CEAS Aeronautical Journal, Vol. 7, No. 1, 2016, pp. 107-119. doi:10.1007/s13272-015-0169-9 
[5] Müller, S., Manzey, D., Schreiter, K., and Luckner, R., "Implementing Energy Status in Head-Down Cockpit Displays: Impact of Augmented Energy Information on Pilot's Performance," 59th Annual Meeting of the Human Factors and Ergonomics Society, Human Factor and Ergonomics Soc., Santa Monica, CA, 2015, pp. 926-930. doi:10.1177/1541931215591270

[6] Schreiter, K., Müller, S., Luckner, R., and Manzey, D., "Verbesserung von Flugpräzision und Arbeitsbeanspruchung bei manuellen RNPAnflügen durch Vorgaberegler und Anzeigen für den Energiewinkel (nxControl)," D. Luft- und Raumfahrtkongress, Deutsche Gesellschaft für Luft- und Raumfahrt, Bonn, 2016, urn:nbn:de:101:1201610283560

[7] International Standard Organisation (ISO), "Flight DynamicsConcepts, Quantities and Symbols-Part 1: Aircraft Motion Relative to the Air, ISO1151-1," 1988.

[8] Oppenheimer, M. W., Doman, D. B., and Bolender, M. A., "Control Allocation for Overactuated Systems," 14th Mediterranean Conference on Control and Automation, IEEE Publ., Piscataway, NJ, 2006. doi:10.1109/MED.2006.328750

[9] Joos, H., Bals, J., Looye, G., Schnepper, K., and Varga, A., "A MultiObjective Optimisation-Based Software Environment for Control Systems Design," Proceedings of the IEEE International Symposium on Computer Aided Control System Design, IEEE Publ., Piscataway, NJ, 2002, pp. 7-14.

doi:10.1109/CACSD.2002.1036921

[10] Tobie, H., Elliot, E., and Malcom, L., "A New Longitudinal Handling Qualities Criterion," Proceedings of the 18th Annual National Aerospace Electronics Conference, IEEE Publ., Piscataway, NJ, 1966, pp. 93-99.

[11] Amelink, M. H., Mulder, M., Paassen, V., and Flach, J., "Theoretical Foundations for a Total Energy-Based Perspective Flight-Path Display," International Journal of Aviation Psychology, Vol. 15, No. 3, 2005, pp. 205-231. doi:10.1207/s15327108ijap1503_1
[12] Lambregts, T., Rademaker, R., and Theunissen, E., "A New Ecological Primary Flight Display Concept," Proceedings of the 2008 IEEE/AIAA 27th Digital Avionics Systems Conference, IEEE Publ., Piscataway, NJ, 2008, pp. 4.A.1-1-4.A.1-20. doi:10.1109/DASC.2008.4702820.

[13] Blaye, P. L., Roumes, C., Fornette, M.-P., and Valot, C., "Head Up Displays Symbology (HUD): Pre Normative Study for DGAC/SFACT," Tech. Rept. DCSD TR2/05007, Onera, Paris, June 2002.

[14] Artini, F., Demortier, J.-P., and Bouchet, C., and Airbus France, "Flight Control Indicator Displaying the Aircraft's Thrust Information," U.S. Patent, US 7636617 B2, 2009.

[15] Wyatt, I. S., and Honeywell International Inc., "Aircraft Display with Potential Thrust Indicator,” U.S. Patent, US 6262674 B1, 2001.

[16] TU Berlin, Department of Flight Mechanics, Control, and Aeroelasticity, "SEPHIR-Simulator for Educational Projects and Highly Innovative Research," 2014, http:/www.fmra.tu-berlin.de/ menue/forschung/ausstattung/sephir/ [retrieved 15 Aug. 2017].

[17] Austro Control GmbH, "Instrument Approach Procedure Salzburg RNAV (RNP) RWY 33, OWS AD 2.24-6-6," March 2016, http://eaip. austrocontrol.at/lo/161111/Charts/LOWS/ LO_AD_2_LOWS_24-6-6_en.pdf [retrieved 16 Aug. 2017].

[18] Hart, S., and Staveland, L., "Development of NASA-TLX (Task Load Index): Results of Empirical and Theoretical Research," Human Mental Workload, edited by P. A. Hancock, and M. Meshkati, Elsevier NorthHolland, Amsterdam, pp. 139-183, https://www.elsevier.com/books/ human-mental-workload/meshkati/978-0-444-70388-0.

[19] Byers, J. C., Bittner, A. C., and Hill, S. G., "Traditional and Raw Task Load Index (TLX) Correlations: Are Paired Comparisons Necessary?' Advances in Industrial Ergonomics and Safety, edited by A. Mital, Taylor \& Francis, London, 1989, pp. 481-485.

[20] Šidák, Z., "Rectangular Confidence Regions for the Means of Multivariate Normal Distributions," Journal of the American Statistical Association, Vol. 62, No. 318, 1967, pp. 626-633. doi:10.1080/01621459.1967.10482935 